\title{
The social network index and its relation to later- life depression among the elderly aged $\geq 80$ years in Northern Thailand
}

This article was published in the following Dove Press journal:

Clinical Interventions in Aging

8 August 2016

Number of times this article has been viewed

\author{
Myo Nyein Aung' \\ Saiyud Moolphate 2 \\ Thin Nyein Nyein Aung ${ }^{3}$ \\ Chitima Katonyoo ${ }^{2}$ \\ Songyos Khamchai ${ }^{4}$ \\ Pongsak Wannakrairot ${ }^{1}$ \\ 'Faculty of Medicine, Chulalongkorn \\ University, Bangkok, Thailand; \\ ${ }^{2}$ Department of Public Health, \\ Chiang Mai Rajabhat University, \\ Chiang Mai, Thailand; ${ }^{3}$ Department \\ of Public Health, Juntendo University \\ School of Medicine, Tokyo, Japan; \\ ${ }^{4}$ Chiang Mai Provincial Health Office, \\ Chiang Mai, Thailand
}

Background: Having a diverse social network is considered to be beneficial to a person's well-being. The significance, however, of social network diversity in the geriatric assessment of people aged $\geq 80$ years has not been adequately investigated within the Southeast Asian context. This study explored the social networks belonging to the elderly aged $\geq 80$ years and assessed the relation of social network and geriatric depression.

Methods: This study was a community-based cross-sectional survey conducted in Chiang Mai Province, Northern Thailand. A representative sample of 435 community residents, aged $\geq 80$ years, were included in a multistage sample. The participants' social network diversity was assessed by applying Cohen's social network index (SNI). The geriatric depression scale and activities of daily living measures were carried out during home visits. Descriptive analyses revealed the distribution of SNI, while the relationship between the SNI and the geriatric depression scale was examined by ordinal logistic regression models controlling possible covariants such as age, sex, and educational attainment.

Results: The median age of the sample was 83 years, with females comprising of $54.94 \%$ of the sample. The participants' children, their neighbors, and members of Buddhist temples were reported as the most frequent contacts of the study participants. Among the 435 participants, $25 \%$ were at risk of social isolation due to having a "limited" social network group (SNI 0-3), whereas 37\% had a "medium" social network (SNI 4-5), and 38\% had a "diverse" social network (SNI $\geq 6$ ). The SNI was not different among the two sexes. Activities of daily living scores in the diverse social network group were significantly higher than those in the limited social network group. Multivariate ordinal logistic regression analysis models revealed a significant negative association between social network diversity and geriatric depression.

Conclusion: Regular and frequent contact with various social contacts may safeguard common geriatric depression among persons aged $\geq 80$ years. As a result, screening those at risk of social isolation is recommended to be integrated into routine primary health care-based geriatric assessment and intervention programs.

Keywords: aging, gerontology, psychogeriatrics, sociology of aging, community, Southeast Asia, Chiang Mai

\section{Introduction}

Social ties are important for a persons' physical and mental well-being. ${ }^{1}$ Since the late 1900s research has been carried out to investigate the impact of social ties on health and lifespan. ${ }^{2,3}$ Cohen et $\mathrm{l}^{2}$ reported the preventive effect of social network diversity on illnesses such as the common cold. Recent epidemiological studies have shown that socially active people are less likely to develop noncommunicable diseases such as diabetes and metabolic syndrome and are likely to live longer than (c)
hereby accept the Terms. Non-commercial uses of the work are permitted without any furcther permission from Dove Medical Press Limited, provided the work is properly attributed. For permission for commercial use of this work, please see paragraphs 4.2 and 5 of our Terms (https://www.dovepress.com/terms.php). 
their nonactive counterparts. ${ }^{4-6}$ Therefore, being connected in social networks may allow us to lead a healthy life by its salutary and supportive social impacts.

The gift of a socially active life is not experienced by all elderly people whose contact list fades away with years of survival. Nowadays, due to the trend of population aging, global societies are seeing more and more people living into their 80 s and $90 \mathrm{~s}^{7}$ For a significant minority of these people, however, a common negative aspect of old age is that of social isolation. ${ }^{89}$ A recent national survey in Malaysia, a neighboring country to the study site, reported that $48.6 \%$ of old persons were at risk of social isolation and that the oldest old persons were at higher risk. ${ }^{10,11}$ Research in Korea, Singapore, and the People's Republic of China also investigated social connectedness of elderly people in each society. Although different instruments were applied in those studies, they reported similarly that people in the bracket of oldest age are liable to social isolation. ${ }^{11-15}$

Research exploring the nature and diversity of elderly people's social contacts is therefore a necessity, particularly given the unique social and cultural contexts within different countries. In 2010, Thailand was home to 8.5 million elderly people aged $\geq 60$ years and now stands as the fastest population-aging country in Southeast Asia. ${ }^{16,17}$ In 2006, people aged $\geq 60$ years comprised $11.3 \%$ of the Thai population, a figure which is estimated to reach $29.8 \%$ by $2050 .{ }^{18,19}$ People aged $\geq 80$ years, meanwhile, constituted $11.5 \%$ of the elderly population, ${ }^{17}$ a figure which is similarly estimated to increase to $23.6 \%$ by $2050 .{ }^{18}$ Although the impact of social networks has been investigated in a wide range of contexts globally, the types and diversity of social contacts in the oldest age group of Thai society is still scant.

An increasingly common problem affecting the elderly is depression. This problem may affect as many as $20 \%$ of those in the oldest age bracket. ${ }^{20}$ However, psychogeriatric research, investigating, for example, the prevalence of depression among the elderly in a country like Thailand, where psychogeriatric services are not yet in place, is still limited. An inverse relation between social network and geriatric depression was reported in a recent study in the US. ${ }^{21}$ Similar studies, however, are still necessary for Thailand and the Southeast Asian context to find out how social networks influence geriatric depression, in particular among people aged $\geq 80$ years.

The current study, therefore, measuring the social network index (SNI) using a quantitative approach aimed to reveal the types of social ties experienced by Thais in the oldest age bracket and assess how their SNI relates to geriatric depression. The findings of the current study seek to highlight the importance of social networks in active aging and may provide insights into ways to preserve the social network diversity of the elderly, resulting in their improved physical and mental well-being.

\section{Design and methods Ethical approval}

This study was approved by Chiang Mai Provincial Health Office, Ethics Review Committee (EC-CMPHO 2/56). The participants were interviewed and written informed consent was obtained.

\section{Data collection procedures}

A community-based, cross-sectional study was conducted in Chiang Mai Province, Northern Thailand. The study sample comprised of 435 people aged $\geq 80$ years at the time of the survey. All were community residents. The sample size was calculated to ensure the power of the finding with a $95 \%$ confidence interval and to represent the population of 10,461 people aged $\geq 80$ years living in the Chiang Mai Province. A multistage sampling approach was applied, taking into account the geographical area, population size, and whether the districts were rural or urban.

Multistage sampling was designed to obtain a representative sample in a stratified proportionate approach. There are totally 25 districts in Chiang Mai Province. A large district, a medium district, and a small district were selected in the first stage of sampling through stratified random sampling approach applying population size to form stratum (Table 1).

From each selected district, one subdistrict was selected by applying simple random sampling. From each subdistrict, participants were sampled in consecutive villages starting from village number one, until a defined number of participants were recruited.

The district Muang Chiang Mai is the main city of Chiang Mai province. Comprising of 16 subdistricts, its population size is comparable to the population of a southern part, northern part, and middle part of the province (Table 1). Therefore, three subdistricts were selected randomly from the 16 subdistricts of Muang Chiang Mai (Table 1). Finally, the sample represented Chiang Mai Province by selecting a total of ten districts. Researchers traveled to villages in twelve subdistricts to reach 435 participants and collected data through interviews (Table 1).

The interviewer administered the questionnaires, and measurement was carried out during home visits in ten districts of Chiang Mai Province. Public health officers who were well trained to interview elderly people conducted data 
Table I Multistage sampling approach to get the sample population $\geq 80$ years of age in Chiang Mai Province, Thailand

\begin{tabular}{llll}
\hline Sampling approaches & $\begin{array}{l}\text { Stratified random } \\
\text { sampling (Stage I) }\end{array}$ & $\begin{array}{l}\text { Simple random } \\
\text { sampling (Stage 2) }\end{array}$ & $\begin{array}{l}\text { Number of participants from } \\
\text { consecutive villages (Stage 3) }\end{array}$ \\
\hline From nine districts in southern part & A large district & I subdistrict & 55 \\
& A medium district & I subdistrict & 37 \\
& A small district & I subdistrict & 18 \\
From eight districts in northern part & A large district & I subdistrict & 55 \\
& A medium district & I subdistrict & 37 \\
From seven districts in middle part & A small district & I subdistrict & 18 \\
& A large district & I subdistrict & 55 \\
From I6 subdistricts in Muang, & A medium district & I subdistrict & 37 \\
main Chiang Mai city & A small district & I subdistrict & 18 \\
& & I subdistrict & 35 \\
\hline
\end{tabular}

Note: The table explains how the multistage sampling approach was applied to collect a representative sample of 435 participants aged 80 years and older, within Chiang Mai Province.

collection from May to July 2014. The response rate was $97.7 \%$ among those who were interviewed.

\section{Research instrument and measurement} Independent and dependent variables

The SNI was calculated from the responses to twelve questions. ${ }^{2}$ This index counts the number of social roles in which the respondent has regular contact, at least once every 2 weeks, with at least one person: spouse, parents, their children and children-in-law, close relatives, close friends, religious members (such as church or temple), classmates, teachers and students in adult education; coworkers or colleagues: employee or employer, neighbors, volunteer networks; and others organizations: social clubs, recreational groups, trade unions, commercial groups, professional organizations, and clubs for the elderly. Hereafter, such regular contact will be referred as "active social contact" in the "Results" and "Discussion" sections.

The maximum SNI score is 12 . Three categories of social network diversity were formed based on the SNI score: SNI 1-3 represents a "limited" social network, 4-5 as a "medium" social network, and SNI $\geq 6$ as a "diverse" social network. SNI is freely accessible in Measurement Instrument Database for the Social Sciences. ${ }^{2,22}$ The instrument was transculturally translated from its original English version into Thai so as to maximize the participants' comprehension and ease of use and tested in a pilot study prior to actual data collection.

To measure depression a 30-item long form geriatric depression scale (GDS) was applied, which had been validated and adopted for the Thai population..$^{23,24}$ Reliability of GDS scale was tested in a pilot sample of 30 people. The data collectors were well trained prior to carrying out the study. The Cronbach's alpha rating GDS in the current study population was 0.8 .

\section{Controlled variables}

Barthel's activities of daily living (ADL) index was applied to measure the participants' daily activity. ${ }^{25,26}$ The ADL scores were categorized into dependent group (ADL < 14) and independent group (ADL 14-20). ${ }^{27}$ The participants' self-rated health status was investigated using a 5-point scale, with responses including "very bad," "bad," "reasonable," "good," and "very good." The responses "bad" and "very bad" were categorized as "poor health status," while the others were categorized as "good health status." Memory assessment applied a single-item assessment tool. Long-term memory was assessed by asking "Can you remember the events that happened years ago?" and short-term memory was assessed by asking participants "Do you remember what you ate for breakfast this morning?" 26

Disability assessment applied long-term disability and short-term disability assessment. ${ }^{27,28}$ Participants were asked two questions to detect long-term disability: "Have you had any condition or health problem for 6 months or longer?" and "Does it prevent or limit you in the kind or amount of activity you can do?"28 A positive response to both of these questions was defined as long-term disability. Participants were asked two questions to detect short-term disability: "Have you had any condition or health problem within the last two weeks?" and "Did it prevent or limit you in the kind or amount of activity you can do?"

\section{Statistical analysis}

The distribution and types of social ties were analyzed through descriptive analysis. The difference between geriatric parameters such as ADL, GDS, disability, and memory loss among the three groups of social network diversity was analyzed applying the Kruskal-Wallis and chi-squared 
tests. Categorization of data such as ADL and GDS followed existing standard references. ${ }^{16,29}$

To examine the hypothesis whether increase in SNI would significantly decrease the rank of GDS, the ordinal logistic regression analysis was applied. The dependent variable GDS was regarded as the ordinal variable. Three models of ordinal logistic regression analyses were applied to test the association between the dependent and independent variables. Various covariates were controlled in three regression models. $P$-value $<0.05$ was considered as statistically significant with a 95\% confidence interval. Stata version 11 (Stata Corporation, College Station, TX) was used for data analysis.

\section{Results}

The median age of the sample was 83 years. More than half (54.94\%) of the sample (435 persons) were female. The average SNI, in terms of social network diversity, was $4.9 \pm 1.9$ (mean \pm standard deviation [SD]). The median number of people in the contact networks was 15 , with a range of 5-21. Categorizing the participants' SNI, 25\% fell into the "limited" social network group (SNI 0-3), 37\% into the "medium" network group (SNI 4-5), and 38\% into the "diverse" social network group ( $\mathrm{SNI} \geq 6$ ). Table 2 shows the characteristics of the participants according to the different categories of SNI. The SNI did not differ between males and females.

The participants' most frequent regular contacts were with their children or their neighbors, with religious society members as their third most active social tie (Figure 1). Contact with close friends and close relatives, meanwhile, represented active social ties for less than half of the participants. Only a third of the participants reported their spouse as an active social contact: $52 \%$ of the males reported this as an active social contact, compared with only $29 \%$ of the females $(P<0.001$, chi-squared).

The ADL scores differed significantly among the three different SNI groups (Table 2). There was a significant difference in age across the three groups of SNI. The proportion of females was uniform and more than the males in all groups. The elderly in the "diverse" social network group whose SNI $\geq 6$ had the highest ADL score. The GDS score of this group was the lowest. Approximately $10.34 \%$ of the participants were suffering from depression: $80 \%$ from "mild" depression; 16\% from "moderate" depression; and $4 \%$ from "severe" depression (Table 2). The proportion of people with a long-term disability and those with a longterm memory loss were significantly higher among elderly

Table 2 Characteristic distribution of senior elderly people aged $\geq 80$ years in categories of social network index

\begin{tabular}{|c|c|c|c|c|c|c|}
\hline \multirow[t]{3}{*}{ Participants' characteristics } & \multirow[t]{3}{*}{ Total } & \multicolumn{3}{|c|}{ Social network } & \multirow[t]{3}{*}{$P$-value } & \multirow[t]{3}{*}{ Test } \\
\hline & & Limited & Medium & Diverse & & \\
\hline & & SNI I-3 & SNI 4-5 & $\mathbf{S N I} \geq 6$ & & \\
\hline \multicolumn{7}{|l|}{ Sociodemographic } \\
\hline Number of participants (\%) & $435(100)$ & $108(24.83)$ & I6I (37.0I) & $166(38.16)$ & & \\
\hline Age, years (mean \pm SD) & $83.8 \pm 3.5$ & $84.7 \pm 4.0$ & $84.0 \pm 3.7$ & $83.1 \pm 2.8$ & 0.017 & K \\
\hline \multicolumn{7}{|l|}{ Sex, n (\%) } \\
\hline Female & $239(54.94)$ & $66(6 I .11)$ & $87(54.04)$ & $86(5 I .8 I)$ & 0.31 & $\mathrm{C}$ \\
\hline Male & $196(45.06)$ & $42(38.89)$ & $74(45.96)$ & $80(48.19)$ & & \\
\hline \multicolumn{7}{|l|}{ Obtained formal education, $\mathrm{n}(\%)$} \\
\hline Yes & $346(79.54)$ & 75 (69.44) & I $27(78.88)$ & I 44 (86.75) & 0.002 & $\mathrm{C}$ \\
\hline No & $89(20.46)$ & $33(37.56)$ & $34(2|| 2)$. & $22(13.25)$ & & \\
\hline \multicolumn{7}{|l|}{ Residence, n (\%) } \\
\hline Urban district & $125(28)$ & $40(37.04)$ & $43(26.7 \mathrm{I})$ & $42(25.30)$ & 0.086 & $\mathrm{C}$ \\
\hline Rural district & $310(71.26)$ & $68(62.96)$ & II 8 (73.29) & $124(74.70)$ & & \\
\hline \multicolumn{7}{|l|}{ Aging parameters } \\
\hline Activity of daily living (mean $\pm S D$ ) & $18.9 \pm 2.7$ & $17.6 \pm 4.4$ & $19.1 \pm 2$ & $19.4 \pm 0.9$ & 0.006 & K \\
\hline \multicolumn{7}{|l|}{ Geriatric parameters } \\
\hline Geriatric depression (mean \pm SD) & $6.2 \pm 4.6$ & $7.35 \pm 5.17$ & $6.5 \pm 4.5$ & $5.19 \pm 4.2$ & $<0.001$ & $\mathrm{~K}$ \\
\hline Persons with long-term disability, n (\%) & $74(17.01)$ & $26(24.07)$ & $29(18.01)$ & $19(11.45)$ & 0.023 & $\mathrm{C}$ \\
\hline Persons with short-term disability, n (\%) & $84(19.31)$ & $13(12.04)$ & $33(20.50)$ & $38(22.89)$ & 0.075 & $\mathrm{C}$ \\
\hline Persons with long-term memory loss, n (\%) & $112(25.75)$ & $32(29.63)$ & $44(27.33)$ & $36(21.69)$ & 0.287 & $\mathrm{C}$ \\
\hline Persons with short-term memory loss, $\mathrm{n}(\%)$ & $59(13.56)$ & $19(17.59)$ & $27(16.77)$ & $13(7.83)$ & 0.023 & $\mathrm{C}$ \\
\hline Self-impression of health & & & & & 0.514 & $\mathrm{C}$ \\
\hline Persons with poor health status, $\mathrm{n}(\%)$ & $5 \mathrm{I}(\mathrm{II.72)}$ & $16(14.81)$ & $17(10.56)$ & $18(10.84)$ & & \\
\hline Persons with good health, n (\%) & $384(88.28)$ & $92(85.19)$ & I44 (89.44) & $148(89.16)$ & & \\
\hline
\end{tabular}

Abbreviations: $\mathrm{C}$, chi-squared test; $\mathrm{K}$, Kruskal-Wallis test; SD, standard deviation; SNI, social network index. 


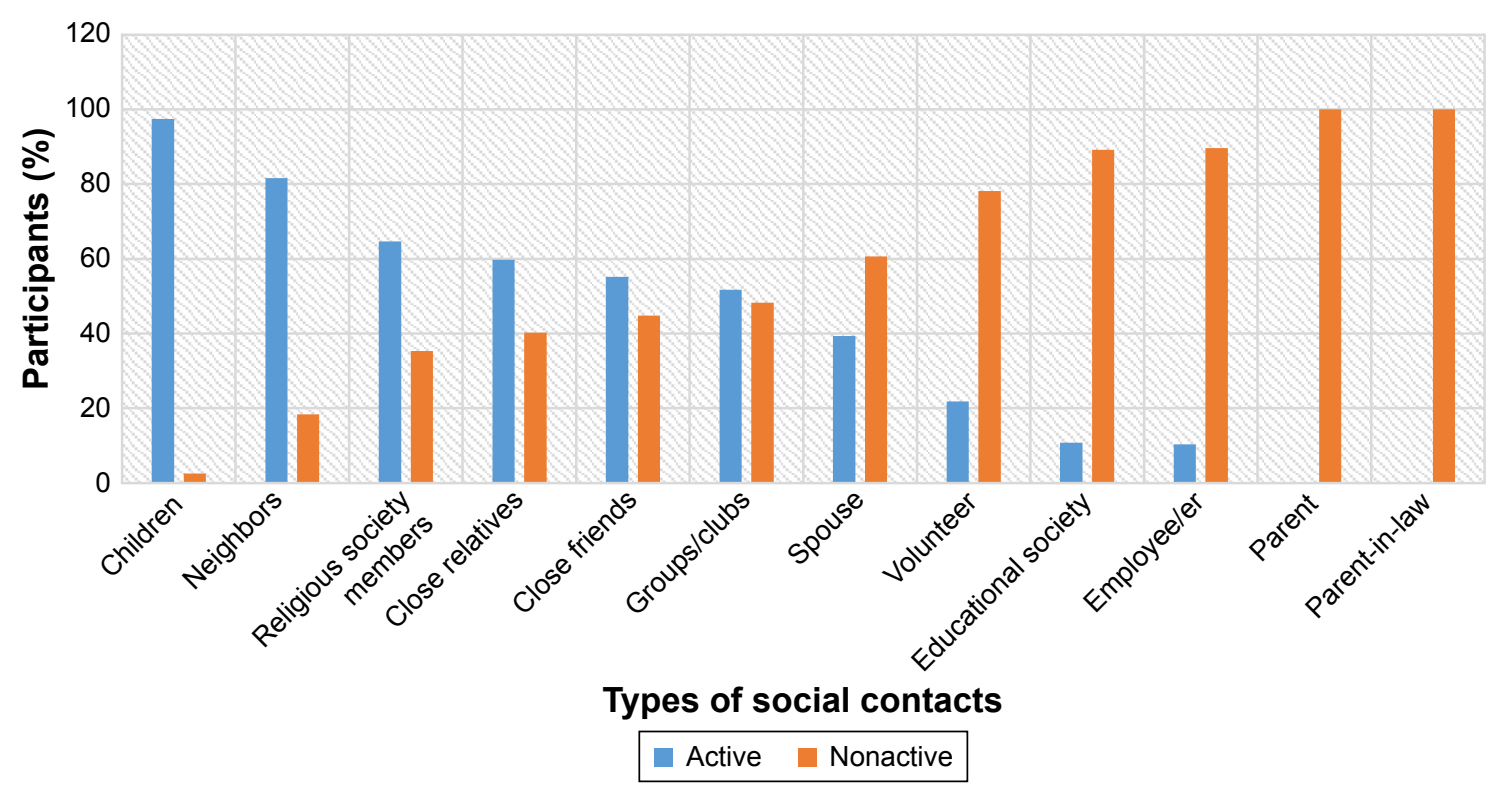

Figure I Distribution of active social network of elderly people aged $\geq 80$ years in Chiang Mai, Northern Thailand, 2014.

seniors with limited social network group than in medium and diverse social network groups.

The level of GDS declined with increasing SNI (Table 2). SNI was significantly associated with GDS in all three models of ordinal logistic regression analyses (Table 3 ). Covariates in the model 1 were SNI, age, sex, and educational attainment; in model 2 were SNI, age, sex, educational attainment, self-impression of health, and dependency; in model 3 were SNI, age, sex, educational attainment, self-impression of health, dependency, and short-term and long-term memory loss (Table 3). The higher the score in the SNI (reflecting a more diverse social network), the lower the GDS score (reflecting lower geriatric depression).

Moreover, participants' good self-rated health status indicated a likely lower level of geriatric depression $(\beta-1.03$, $P<0.001$ ), whereas those with long-term memory loss and short-term memory loss were more likely to suffer from geriatric depression (Table 3).

\section{Discussion}

This study is of particular value because few studies have conducted empirical research into the social network diversity of people aged $\geq 80$ years. ${ }^{30}$ It has revealed common patterns of the social networks among the oldest age group in the Northern Thailand context (Figure 1). Many of the participants have lost their closest ties, such as their spouse and their friends. Hence, those who survive longer than their national life expectancy and enter their 80s and 90s may experience drastic changes in their social network. More than a quarter of the participants in the current study belonged to a very limited social network, SNI $<3$. They were at high risk of social isolation, a health problem overlooked in many communities despite its negative impact on life expectancy, morbidity, and quality of life. ${ }^{31}$ The findings of the current study, therefore, suggest integrating screening for social isolation as part of primary health care-based geriatric assessment.

The majority of participants ranked their children as their most frequently contacted social tie (Figure 1). This highlights the importance of families within the social network of the elderly. In Thai culture, parents, children, and grandchildren traditionally live together, and many elderly people retain a role of caregiver to their grandchildren within these intergenerational families. ${ }^{32}$ Moreover, a recent study reported that such intergenerational families are common to countries such as Thailand, Myanmar, and Vietnam. ${ }^{32}$ Many children may migrate to work outside their native province, for example, for career development, yet they can keep in touch with their parents due to the increasing use of mobile phones, as well as improved road access and transportation in Thailand. ${ }^{33}$

Neighbors were ranked second by the participants among their most frequent contacts. As reported by Basto et al, ${ }^{34}$ neighbors represent an ecological asset for elderly community residents, although this may not be the case for nursing home residents. ${ }^{35}$ Neighborhood hospitality also depends on how urban the location of a community is. Communities in metropolitan Bangkok and traditional communities in Chiang Mai may be rather different. All participants in the current study were community residents living in the Chiang Mai Province, Northern Thailand. This then, may reflect the traditional culture and society unique to Northern Thailand. 
Very few participants retained occupational or educational networks (Figure 1). After retirement, there is less of a role for elderly people within the labor market and occupational networks. ${ }^{8}$ However, aging people still have other possible active social roles within their communities. The lack of formal working hours coupled with a likely more relaxed daily life may permit the elderly to engage more in social volunteering and religious activity. Notably, $>60 \%$ of the participants reported their active contact as a member of the temple. Given that all of the participants were Buddhist, this finding is concurrent with that of another recent study in Thailand which reported that involvement in the Buddhist social network positively contributes to the functional health of the elderly. ${ }^{36}$

Furthermore, more than half of the participants reported being in contact with a group or club. This could be the result of clubs especially for the elderly which have recently been established in a number of districts in Thailand. These clubs have become popular opportunities for the elderly to get together and socialize. In contrast, less than a quarter of participants reported being involved in volunteer work or regularly talked with people in volunteering activities. This finding is very similar to that of a UK study in which around $20 \%$ of the elderly, aged 75 years and older, reported participation in UK volunteering activities. ${ }^{36}$

This study also assessed, in a characteristic sample, the relationship of the participants' SNI to their level of geriatric depression. The self-impression of health and long-term memory loss had a significant association with depression. After adjusting several important covariates, such as demographics (age, sex, and educational attainment), health status (dependency and self-impression of health), and cognitive decline (short-term and long-term memory loss), there was a significant negative association between social network diversity and geriatric depression. Therefore, being in regular and frequent contact with various social contacts may prevent common geriatric depression among those aged $\geq 80$ years (Table 3 ).

The relation between geriatric depression and social network isolation has been reported in different studies with variable results. ${ }^{21,31,37}$ These inconsistent findings are probably due to various social networks and their impacts in different cultural contexts. However, the findings of earlier reports in other parts of Thailand are consistent with the findings of the current study despite the different methodological approaches applied. Thanakwang et $\mathrm{a}^{38}$ reported the positive social impact of friends and families on the psychological well-being of elderly Thais. Sasiwongsaroj et al, ${ }^{36}$ meanwhile, 
reported that elderly Thais involved in the Buddhist social network gained better functional, mental, and social health status as a result of this social network. ${ }^{36}$ The samples in these studies, however, comprised of elderly people aged $>60$ years, whereas the median age within the current study was 83 years. A twenty-year difference in later life may cause drastic changes in social networks, notably with profound loss of friends and colleagues. The current study, therefore, has been able to uncover different typologies of social networking among the oldest bracket of the elderly.

This study may have some limitations. The way social network diversity was measured was based on the number and frequency of contact. The quality of the relationship in each social tie could not be known using this approach, however. Moreover, the relation between variables, tested in a cross-sectional study, may require future longitudinal study and analysis. Despite these shortcomings, it is hoped that public health and long-term care programs will benefit from the findings of the present study. It is considered that the study's descriptive and analytical findings can provide a practical impetus to design evidence-based intervention programs in the Thai setting and more broadly in that of Southeast Asia, which will preserve and promote the social networks of the elderly, in doing so serving to reduce the problem of geriatric depression.

\section{Conclusion}

In the setting of the current study, children, neighbors, and the Buddhist temple members have been reported as the most likely resources of social network for those in the oldest bracket of the elderly. There is still ample opportunity, however, for this group to strengthen their social ties by engaging in volunteer activity and clubs for the elderly. Moreover, the diversity of their social networks may serve to prevent the problem of depression. Therefore, future research should devise community-based intervention to promote the social networks of those in the oldest age bracket, which may lead the elderly to enjoy active aging and living, in turn positively impacting upon their mental health.

\section{Acknowledgments}

The authors acknowledge Rachadapisek Sompote Fund for Senior Postdoctoral Fellowship, Chulalongkorn University, which supported the lead author. The contribution of Wantanee Chawapong, Panida Sennun, Waraporn Wanchaitanawong, Wittaya Tanaree, Mujjarin. Pangsiri, Kanyapat Chaeye, Jitnatee Richie, Naiyana Metha, Nitcha Kongin, Pikul Tanamas, and Surasing Wisarutrat for the project and data collection is well acknowledged. Paisan Thanyawinichkul and Amorn Nondasuta, Chiang Mai provincial health office, are heartily acknowledged for their support to the study. Margot Pambrun and Than Htike Aung are acknowledged for their help to edit the final proof. Without the contribution of health centers, health volunteers, and Pajaree Yenbutra, the WHO collaborating center for medical education, Faculty of Medicine, Chulalongkorn University, this work may not have been possible.

\section{Disclosure}

The authors report no conflicts of interest in this work.

\section{References}

1. Kawachi I, Berkman LF. Social ties and mental health. JUrban Health. 2001;78(3):458-467.

2. Cohen S, Doyle WJ, Skoner DP, Rabin BS, Gwaltney JM Jr. Social ties and susceptibility to the common cold. JAMA. 1997;277(24): 1940-1944.

3. Berkman LF, Syme SL. Social networks, host resistance, and mortality: a nine-year follow-up study of Alameda County residents. Am J Epidemiol. 1979;109(2):186-204.

4. Kreibig SD, Whooley MA, Gross JJ. Social integration and mortality in patients with coronary heart disease: findings from the Heart and Soul Study. Psychosom Med. 2014;76(8):659-668.

5. Yang YC, Li T, Ji Y. Impact of social integration on metabolic functions: evidence from a nationally representative longitudinal study of US older adults. BMC Public Health. 2013;13(1):1210.

6. Hilding A, Shen C, Östenson CG. Social network and development of prediabetes and type 2 diabetes in middle-aged Swedish women and men. Diabetes Res Clin Pract. 2015;107(1):166-177.

7. World Health Organization. Aging. Geneva: World Health Organization; 2015. Available from: http://www.who.int/topics/ageing/en/. Accessed September 29, 2015

8. Llewellyn A, Agu L, Mercer D. Sociology for Social Workers. 2nd ed. Cambridge: Polity; 2015.

9. Shimada K, Yamazaki S, Nakano K, Ngoma AM, Takahashi R, Yasumura S Prevalence of social isolation in community-dwelling elderly by differences in household composition and related factors: from a social network perspective in urban Japan. J Aging Health. 2014;26(5):807-823.

10. Ibrahim R, Abolfathi Momtaz Y, Hamid TA. Social isolation in older Malaysians: prevalence and risk factors. Psychogeriatrics. 2013;13(2): 71-79.

11. Momtaz YA, Ibrahim R, Hamid TA, Yahaya N. Sociodemographic predictors of elderly's psychological well-being in Malaysia. Aging Mental Health. 2011;15(4):437-445.

12. Li T, Zhang Y. Social network types and the health of older adults: exploring reciprocal associations. Social Sci Med (1982). 2015;130:59-68.

13. Lim LL, Kua EH. Living alone, loneliness, and psychological wellbeing of older persons in singapore. Curr Gerontol Geriatr Res. 2011; 2011:673181.

14. Park S, Smith J, Dunkle RE. Social network types and well-being among South Korean older adults. Aging Mental Health. 2014;18(1):72-80.

15. Momtaz YA, Haron SA, Ibrahim R, Hamid TA. Social embeddedness as a mechanism for linking social cohesion to well-being among older adults: moderating effect of gender. Clinical Interv Aging. 2014;9:863-870.

16. Chiang Mai Procincial health office:Chiang mai population by sex and age. [webpage on the Internet]. 2014. Available from: http://cmi.hdc. moph.go.th/hdc/reports/report.php?source=formated/pop_sex_age. php\&cat_id=ac4eed1bddb23d6130746d62d2538fd0\&id=710884bc 8 d16f755073cf194970b064a\#. Accessed July 15, 2016. 
17. Sasat S, Bowers BJ. Spotlight Thailand. Gerontologist. 2013;53(5): 711-717.

18. Hofmeister W. Ageing and politics - consequences for Asia and Europe aging population and health care services in Asain Pacific Region; 2010. Available from: http://www.kas.de/politikdialog-asien/en/ publications/20784/. Accessed November 4, 2015.

19. Bloomberg Visual data: Most rapidly ageing countries; 2015. Available from: http://www.bloomberg.com/visual-data/best-and-worst/ most-rapidly-aging-countries. Accessed September 15, 2015.

20. Hornsten C, Molander L, Gustafson Y. The prevalence of stroke and the association between stroke and depression among a very old population. Arch Gerontol Geriatr. 2012;55(3):555-559.

21. Van Orden KA, Li Y, Podgorski CA, Conwell Y. The association between higher social support and lower depressive symptoms among aging services clients is attenuated at higher levels of functional impairment. Int J Geriatr Psychiatry. 2015;30(10):1085-1092.

22. Cohen S. Social Network Index SNI. Available from: http://www.midss. org/sites/default/files/social_network_index.pdf. Accessed March 20, 2015.

23. Yesavage JA, Brink TL, Rose TL, et al. Development and validation of a geriatric depression screening scale: a preliminary report. J Psychiatr Res. 1982;17(1):37-49.

24. Train The Brain Forum Committee: Thai Geriatric Depression ScaleTGDS. Siriraj Hosp Gaz. 1994;46:1-9.

25. Barthel Activities of Daily Living (ADL) Index. Occas Pap R Coll Gen Pract. 1993;59:24.

26. Department of Medical Service, Ministry of Public Health, Thailand. Manual of assessment tool for ederly people; 2014. Available from: http://agingthai.dms.moph.go.th/agingthai/index.php/guidebook/2042557. Accessed July 15, 2016.

27. Jitapunkul S, Kunanusont C, Phoolcharoen W, Suriyawongpaisal P. Health of Thai Elderly. Bangkok:Holistic Publishing Limited. 1999.

28. Jitapunkul S, Kamolratanakul P, Chandraprasert S, Bunnag S. Disability among Thai elderly living in Klong Toey slum. J Med Assoc Thai. 1994; 77(5):231-238.
29. Wiener JM, Hanley RJ, Clark R, Van Nostrand JF. Measuring the activities of daily living: comparisons across national surveys. J Gerontol. 1990;45(6):S229-S237.

30. Godwin M, Pike A, McCrate F, et al. The healthy aged: descriptive analysis by sex of cognitively functioning elderly patients 80 years and older living independently in the community. Can Fam Physician. 2015; 61(3):e142-e147.

31. Nicholson NR. A review of social isolation: an important but underassessed condition in older adults. J Prim Prev. 2012;33(2-3):137-152.

32. Knodel J, Pothisiri W. Intergenerational living arrangements in Myanmar and Thailand: a comparative analysis. J Cross Cult Gerontol. 2015; 30(1):1-20.

33. Adhikari R, Jampaklay A, Chamratrithirong A. Impact of children's migration on health and health care-seeking behavior of elderly left behind. BMC Public Health. 2011;11(1):143.

34. Bastos AM, Faria C, MoreiraE, Morais D, Melo-de-Carvalho JM, Paul MC. The importance of neighborhood ecological assets in community dwelling old people aging outcomes: a study in Northern Portugal. Front Aging Neurosci. 2015;7:156.

35. Drageset J, Kirkevold M, Espehaug B. Loneliness and social support among nursing home residents without cognitive impairment: a questionnaire survey. Int J Nurs Stud. 2011;48(5):611-619.

36. Sasiwongsaroj K, Wada T, Okumiya K, et al. Buddhist social networks and health in old age: a study in central Thailand. Geriatr Gerontol Int. 2015; 15(11):1210-1218

37. Klug G, Lacruz ME, Emeny RT, Hafner S, Ladwig KH, Huber D. Aging without depression: a cross-sectional study. Psychodyn Psychiatry. 2014; 42(1):5-22.

38. Thanakwang K, Ingersoll-Dayton B, Soonthorndhada K. The relationships among family, friends, and psychological well-being for Thai elderly. Aging Mental Health. 2012;16(8):993-1003.
Clinical Interventions in Aging

\section{Publish your work in this journal}

Clinical Interventions in Aging is an international, peer-reviewed journal focusing on evidence-based reports on the value or lack thereof of treatments intended to prevent or delay the onset of maladaptive correlates of aging in human beings. This journal is indexed on PubMed Central, MedLine,

\section{Dovepress}

CAS, Scopus and the Elsevier Bibliographic databases. The manuscript management system is completely online and includes a very quick and fair peer-review system, which is all easy to use. Visit http://www.dovepress. com/testimonials.php to read real quotes from published authors. 